\title{
Integrity of the Actin Cytoskeleton of Host Macrophages is Necessary for Mycobacterial Entry
}

\author{
Aritri Dutta ${ }^{1} \cdot$ Ravi Prasad Mukku $^{1} \cdot$ G. Aditya Kumar $^{1,2} \cdot$ Md. Jafurulla ${ }^{1} \cdot$ Tirumalai R. Raghunand $^{1}$. \\ Amitabha Chattopadhyay ${ }^{1}$ (D)
}

Received: 24 December 2021 / Accepted: 27 January 2022 / Published online: 15 February 2022

(c) The Author(s), under exclusive licence to Springer Science+Business Media, LLC, part of Springer Nature 2022

\begin{abstract}
Macrophages are the primary hosts for Mycobacterium tuberculosis (M. tb), an intracellular pathogen, and the causative organism of tuberculosis (TB) in humans. While $M$. $t b$ has the ability to enter and survive in host macrophages, the precise mechanism of its internalization, and factors that control this essential process are poorly defined. We have previously demonstrated that perturbations in levels of cholesterol and sphingolipids in macrophages lead to significant reduction in the entry of Mycobacterium smegmatis (M. smegmatis), a surrogate model for mycobacterial internalization, signifying a role for these plasma membrane lipids in interactions at the host-pathogen interface. In this work, we investigated the role of the host actin cytoskeleton, a critical protein framework underlying the plasma membrane, in the entry of M. smegmatis into human macrophages. Our results show that cytochalasin D mediated destabilization of the actin cytoskeleton of host macrophages results in a dose-dependent reduction in the entry of mycobacteria. Notably, the internalization of Escherichia coli remained invariant upon actin destabilization of host cells, implying a specific involvement of the actin cytoskeleton in mycobacterial infection. By monitoring the F-actin content of macrophages utilizing a quantitative confocal microscopy-based technique, we observed a close correlation between the entry of mycobacteria into host macrophages with cellular F-actin content. Our results constitute the first quantitative analysis of the role of the actin cytoskeleton of human macrophages in the entry of mycobacteria, and highlight actin-mediated mycobacterial entry as a potential target for future anti-TB therapeutics.
\end{abstract}

\section{Graphical Abstract}

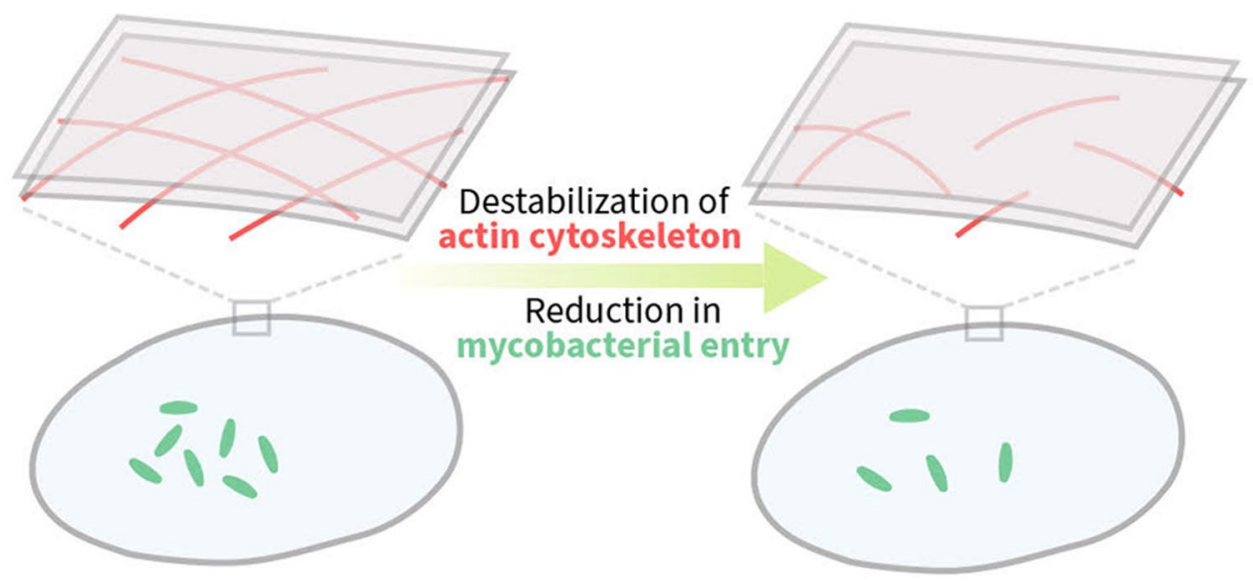

Keywords Actin cytoskeleton $\cdot$ Cytochalasin D $\cdot$ F-actin quantitation $\cdot$ Macrophages $\cdot$ Mycobacterium

Aritri Dutta, Ravi Prasad Mukku and G. Aditya Kumar have equally contributed to this study.

Extended author information available on the last page of the article 


\section{Introduction}

Intracellular pathogens employ diverse strategies to invade and establish infection in host cells, the success of which depends mainly on their utilization of host cellular components and averting host defense responses (Asrat et al. 2014; Bagga and Bouchard 2014; Liu et al. 2017; Thakur et al. 2019). Mycobacterium tuberculosis, the causative organism of tuberculosis (TB) in humans, has been identified to utilize multiple mechanisms for establishing infection (Stanley and Cox 2013; Awuh and Flo 2017; Chaurasiya 2018; Fraga et al. 2018). A comprehensive understanding of these events is crucial in deciphering the processes underlying infection by this deadly pathogen, which remains a major cause of mortality and morbidity worldwide. An estimated 10 million new cases and 1.5 million deaths were reported in 2020 alone, which is further worsened by its opportunistic infection among HIV positive patients and the shortfall in case notifications due to the ongoing COVID-19 pandemic (World Health Organization 2021). Importantly, immediate attention toward designing novel therapeutic strategies to treat this disease has become necessary due to the emergence of multi- and extensively drug-resistant TB (Gandhi et al. 2006; Dye 2009; Keshavjee and Farmer 2012; Lange et al. 2018; Singh et al. 2020; Allué-Guardia et al. 2021).

As a prerequisite to gain access into host cells, mycobacteria interact with and traverse the host cell plasma membrane. It is therefore critical to understand the events and players involved at this first step of mycobacterial entry into its host, which later determines its growth, dissemination and pathogenesis. Targeting this step has the potential advantage of preventing infection early enough, most likely before the manifestation of clinically relevant pathology (El-Etr and Cirillo 2001). Over the years, several key cell surface components of the host and the pathogen have been identified to facilitate efficient host-pathogen interaction. These include a number of candidate receptors on the cell surface of host macrophages, important for the recognition and entry of mycobacteria such as complement receptors, mannose receptor, CD-14, scavenger receptors, DC-SIGN, and Dectin-1 (Ernst 1998; Killick et al. 2013; Awuh and Flo 2017). In this context, we have previously demonstrated a role for host membrane cholesterol and sphingolipids in the entry of mycobacteria into macrophages, by modulating the availability of these lipids using a combination of complementary approaches (Viswanathan et al. 2015, 2018).

The actin cytoskeleton, a dynamic protein network that is involved in cellular processes such as trafficking, motility and signaling, lies beneath the lipid bilayer and has emerged as an integral component in the contemporary model of the plasma membrane (Janmey 1998; Honigmann and Pralle 2016; Chakrabarti et al. 2021). Interestingly, membrane cholesterol and the actin cytoskeleton are believed to exhibit dynamic interplay in terms of their content and organization (Kwik et al. 2003; Sun et al. 2007; Ganguly and Chattopadhyay 2010; Sarkar P, Kumar GA, Shrivastava S, Chattopadhyay A (unpublished observations)). Since the host plasma membrane serves as the first portal of entry for pathogens (Kumar et al. 2016), investigating the role of host actin cytoskeleton in the entry of mycobacteria could prove to be extremely insightful in understanding the mechanistics of this essential process. In the present work, we explored the role of the host actin cytoskeleton in the entry of Mycobacterium smegmatis into human THP-1 macrophages. Since the composition and architecture of their cell surface components are conserved, $M$. smegmatis serves as an effective surrogate model for $M$. tuberculosis in addressing the mechanisms of mycobacterial entry (Sani et al. 2010; Zhang 2013).

In order to explore the role of actin cytoskeleton in the entry of M. smegmatis, we employed cytochalasin D (CD), a potent inhibitor of actin polymerization. Actin is an abundant cytosolic protein found in eukaryotes both as monomeric G-actin and polymeric F-actin, with F-actin being maintained in a dynamic equilibrium with soluble G-actin (Dominguez and Holmes 2011). In vitro studies have shown that $\mathrm{CD}$ stops actin polymerization by binding to the barbed end (the fast growing end) of the actin filament, thereby shifting the equilibrium toward depolymerization (Schliwa 1982; Sampath and Pollard 1991). Our results show that destabilization of the actin cytoskeleton of host macrophages by $\mathrm{CD}$ results in a dose-dependent reduction in the entry of $M$. smegmatis into macrophages. We monitored F-actin content of macrophages utilizing a quantitative confocal microscopy-based technique, previously developed by us (Ganguly et al. 2011). Notably, we observed a close correlation between the entry of mycobacteria into host macrophages with cellular F-actin content, a pointer to the molecular basis for the involvement of actin cytoskeleton in mycobacterial entry. To the best of our knowledge, these results constitute the first quantitative analysis on the role of the actin cytoskeleton of human macrophages in the entry of mycobacteria.

\section{Materials and Methods}

\section{Materials}

$\mathrm{MgCl}_{2}, \mathrm{CaCl}_{2}$, penicillin, streptomycin, gentamycin sulfate, CD, DMSO, 3-(4,5-dimethylthiazol-2-yl)-2,5-diphenyltetrazolium bromide (MTT), NaCl, Phorbol 12-myristate 13-acetate (PMA), Triton X-100 and Tween 80 were obtained from 
Sigma Chemical Co. (St. Louis, MO). Middlebrook 7H9 broth and 7H10 agar were obtained from Becton, Dickinson Difco (Sparks, MD). RPMI-1640 medium and fetal bovine serum were obtained from Gibco/Life Technologies (Grand Island, NY). Alexa Fluor 546 phalloidin was from Molecular Probes/Invitrogen (Eugene, OR). Vectashield ${ }^{\circledR}$ antifade mounting medium containing DAPI was obtained from Vector Laboratories (Burlingame, CA). All chemicals used were of the highest available purity. Water was purified through a Millipore (Bedford, MA) Milli-Q system and used throughout.

\section{Cell Culture}

The human monocyte cell line THP-1 (American Type Culture Collection) was maintained and differentiated as described previously (Tiwari et al. 2012, 2014). Briefly, cells were grown in RPMI-1640 medium supplemented with $2 \mathrm{~g} / \mathrm{l}$ of sodium bicarbonate, $60 \mu \mathrm{g} / \mathrm{ml}$ penicillin, $50 \mu \mathrm{g} / \mathrm{ml}$ streptomycin, $30 \mu \mathrm{g} / \mathrm{ml}$ gentamycin sulfate and $10 \%$ fetal bovine serum in a humidified atmosphere with $5 \% \mathrm{CO}_{2}$ at $37{ }^{\circ} \mathrm{C}$. THP- 1 monocytes were differentiated into macrophages by treatment with $5 \mathrm{ng} / \mathrm{ml}$ PMA for $24 \mathrm{~h}$ and were subsequently grown in PMA-free medium for $48 \mathrm{~h}$, prior to further treatments.

\section{Bacterial Culture}

Mycobacterium smegmatis $\mathrm{mc}^{2} 6$ and E. coli $\mathrm{DH} 5 \alpha$ were cultured as described previously (Viswanathan et al. 2015, 2018). M. smegmatis was grown in Middlebrook 7 H9 broth and 7H10 agar supplemented with albumin dextrose complex (5 g/l BSA, $2 \mathrm{~g} / \mathrm{l}$ glucose and $0.85 \mathrm{~g} / \mathrm{l} \mathrm{NaCl}), 0.5 \%(\mathrm{v} / \mathrm{v})$ glycerol and $0.05 \%(\mathrm{v} / \mathrm{v})$ Tween 80 under shaking conditions at $37^{\circ} \mathrm{C}$. E. coli $\mathrm{DH} 5 \alpha$ was grown in Luria Bertani broth under similar conditions.

\section{Cytochalasin D Treatment of THP-1 Macrophages}

Destabilization of the actin cytoskeleton of THP-1 macrophages was carried out using $\mathrm{CD}$ as described previously (Ganguly et al. 2008, 2011; Roy et al. 2014) with some modifications. Briefly, a stock solution of CD $(2 \mathrm{mM})$ was prepared in DMSO and further concentrations required for treating macrophages were prepared by diluting it in serum-free RPMI-1640 medium. Prior to treatment, macrophages were incubated in serum-free medium for $3 \mathrm{~h}$ at $37^{\circ} \mathrm{C}$, and were then treated with increasing concentrations of $\mathrm{CD}$ for $30 \mathrm{~min}$ at $37^{\circ} \mathrm{C}$ in the same medium. After the treatment, medium was removed to take away excess $\mathrm{CD}$ and macrophages were washed twice with PBS prior to the next steps. During the treatments, the amount of DMSO was always maintained at $<0.5 \%$ (v/v). Treatment of control cells with similar amount of DMSO did not affect cellular morphology.

\section{MTT Viability Assay}

The viability of THP-1 macrophages upon treatment with CD was assessed using an MTT assay as described earlier (Viswanathan et al. 2015) with some modifications. In brief, macrophages were plated at a density of $\sim 2 \times 10^{5}$ in 24 well plates and were treated with $\mathrm{CD}$ as described above. Cells were then washed with serum-free RPMI-1640 medium and were incubated with MTT dissolved in the same medium at a final concentration of $0.4 \mathrm{mg} / \mathrm{ml}$ at $37{ }^{\circ} \mathrm{C}$ for $2.5 \mathrm{~h}$. In live cells, mitochondrial enzymes would reduce the MTT salt to formazan (Vistica et al. 1991), the formazan crystals formed were dissolved in DMSO and the color obtained was measured by absorbance at $540 \mathrm{~nm}$ in a PowerWave XS2 microplate spectrophotometer (BioTek Winooski, VT).

\section{F-Actin Labeling}

Labeling of F-actin in THP-1 macrophages was carried out as described earlier (Ganguly et al. 2011; Roy et al. 2014) with some modifications. Macrophages were plated at a density of $\sim 2 \times 10^{6}$ on glass coverslips and were treated with $\mathrm{CD}$. The macrophages were subsequently washed with PBS containing $1 \mathrm{mM} \mathrm{CaCl}_{2}$ and $0.5 \mathrm{mM} \mathrm{MgCl}$ (buffer A) and were then fixed with $4 \%(\mathrm{w} / \mathrm{v})$ formaldehyde for $10 \mathrm{~min}$. Cells were permeabilized with $0.5 \%$ Triton X-100 (v/v) in buffer A for 6 min. Cells were further washed and stained with Alexa Fluor 546 phalloidin for $1 \mathrm{~h}$ and mounted for subsequent confocal imaging.

\section{Confocal Microscopy and Quantitation of F-Actin}

The effect of CD treatment on actin cytoskeleton in macrophages was assessed by quantitation of F-actin from images acquired using confocal microscopy. Confocal microscopic images of THP-1 macrophages were acquired on an inverted Zeiss LSM 880 confocal microscope (Jena, Germany). F-actin labeled with Alexa Fluor 546 phalloidin (as described above) was imaged by exciting at $543 \mathrm{~nm}$ and collecting emission from $550 \mathrm{~nm}$ to $650 \mathrm{~nm}$. F-actin was quantified using a technique previously developed by us (Ganguly et al. 2011) with some modifications. Briefly, images of $z$-sections were acquired with a $63 \mathrm{x} / 1.4 \mathrm{NA}$ oil immersion objective under 1 airy condition, with a fixed step size of $0.4 \mu \mathrm{m}$. Maximum intensity projections were generated from 11 sections $(\sim 4 \mu \mathrm{m}$ from the base into the cell), and area bound by the projected images was determined manually using the Zen software provided with Zeiss LSM 880 confocal microscope. Images of $z$-sections corresponding to each projected image were processed by fluorescence 
intensity thresholding, followed by the application of a Gaussian filter using Imaris 8.0.0 software (Bitplane AG, Zurich, Switzerland). Contours generated upon joining voxels of equal fluorescence intensity, called iso-surfaces, were generated from $z$-sections. The volumes enclosed by iso-surfaces were normalized to the projected area of cells for each field and used as a quantitative estimate of cellular F-actin levels.

\section{Quantification of Entry of Bacteria Into Macrophages}

Entry of M. smegmatis and E. coli into THP-1 macrophages was quantified by scoring colony forming units (CFU), as described earlier (Tiwari et al. 2012, 2014). Macrophages plated on 6-well plates at a density of $\sim 2 \times 10^{6}$ were infected with exponentially growing $M$. smegmatis or E. coli at a multiplicity of infection (MOI) of 100:1 (bacteria to macrophage). Prior to infection, $M$. smegmatis culture was passed 5-6 times through 261/2 gauge needles to obtain single cell suspensions. Macrophages treated with (or without) $\mathrm{CD}$ were incubated with bacteria for $2 \mathrm{~h}$ and were washed with PBS. Extracellular bacteria were killed by treatment with gentamycin $(50 \mu \mathrm{g} / \mathrm{ml})$ in serum-free RPMI-1640 for $30 \mathrm{~min}$ at $37^{\circ} \mathrm{C}$. After washing at least 3 times with antibiotic-free media, macrophages were lysed with $0.1 \%$ Triton X-100 (v/v). For M. smegmatis, macrophage cell lysates were plated on Middlebrook 7H10 agar upon preparing appropriate dilutions in Middlebrook 7H9 media. In case of E. coli, lysates were plated on Luria Bertani agar after dilutions were made in Luria Bertani broth. To enumerate colony forming unit (CFU) counts, bacterial culture plates were incubated at $37^{\circ} \mathrm{C}$ till visible colonies appeared.

\section{Fluorescence Confocal Microscopy of Macrophages Infected with M. smegmatis}

The effect of CD treatment on the entry of $M$. smegmatis into THP-1 macrophages was visualized by confocal microscopic imaging as described previously (Tiwari et al. 2012, 2014), with some modifications. Briefly, macrophages were plated on glass coverslips at a density of $\sim 2 \times 10^{6}$ and were treated with varying concentrations of $\mathrm{CD}$. Macrophages were then infected with $M$. smegmatis stably expressing the fluorescent protein dsRed2 (strain transformed with pMSP12::dsRed2) (Cosma et al. 2004). Cells were washed, fixed with $4 \%$ formaldehyde (w/v) and mounted using Vectashield ${ }^{\circledR}$ antifade mounting medium containing DAPI. Images of $z$-sections were acquired on an inverted Zeiss LSM 880 confocal microscope (Jena, Germany) with a $63 x / 1.4$ NA oil immersion objective under 1 airy condition, with a fixed step size of $1 \mu \mathrm{m}$. Confocal $z$-sections were then merged to generate maximum intensity projections.

\section{Statistical Analysis}

Significance levels were calculated using Student's twotailed paired $t$-test using Graphpad Prism software (version 4.0; San Diego, CA) with 95\% confidence interval. The correlation between $M$. smegmatis $\mathrm{CFU}$ counts and F-actin content of host macrophages with increasing concentrations of $\mathrm{CD}$ was analyzed using the same software. Plots were generated using OriginPro software, version 8.0 (OriginLab, Northampton, MA).

\section{Results}

\section{Cytochalasin D Induced Alteration of Actin Organization in THP-1 Macrophages}

To explore the role of actin cytoskeleton of human macrophages in the entry of mycobacteria, we treated THP-1 macrophages with $\mathrm{CD}$, a potent inhibitor of actin polymerization. The effect of $\mathrm{CD}$ on the macrophage actin
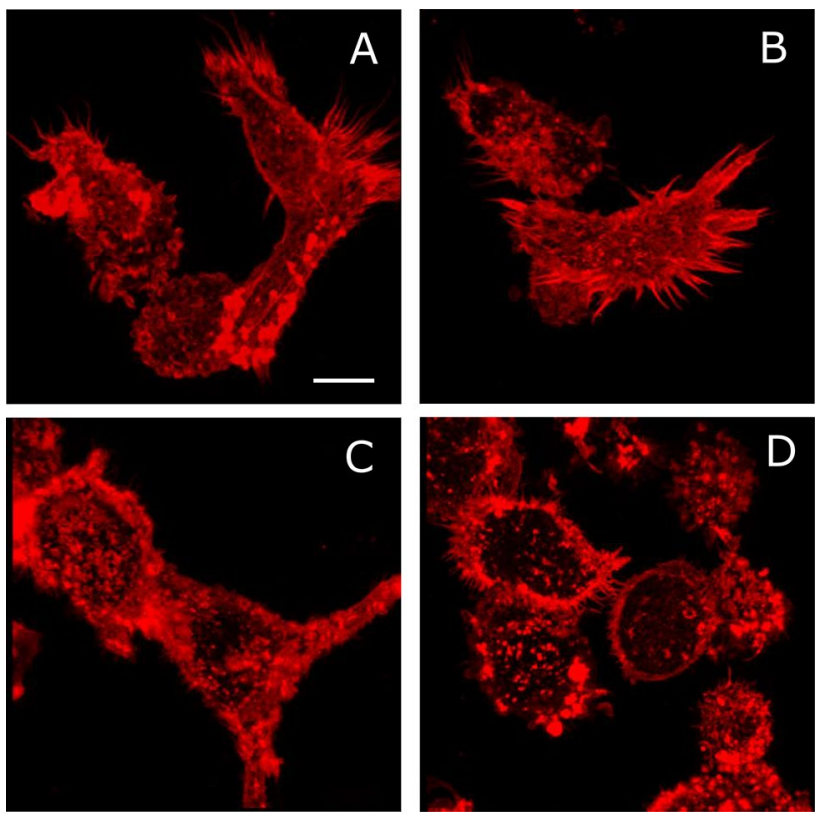

Fig. 1 Actin organization in THP-1 macrophages upon treatment with cytochalasin D. THP-1 macrophages were treated with increasing concentrations of $\mathrm{CD}$ and actin organization was evaluated using confocal microscopy. Alexa Fluor 546 phalloidin was used to stain $\mathrm{F}$-actin in macrophages. Maximum intensity projections obtained by merging 11 sections from the base of the coverslip $(\sim 4 \mu \mathrm{m}$ from the base into the cell) are shown. Representative projected images for control macrophages are shown in panel $(\mathbf{A})$ and the corresponding images for macrophages treated with $2.5 \mu \mathrm{M}, 5 \mu \mathrm{M}$ and $10 \mu \mathrm{M} \mathrm{CD}$ are shown in panels (B-D), respectively. Upon treatment with increasing concentrations of $\mathrm{CD}$, reduction in F-actin filaments and formation of F-actin aggregates were observed. The scale bar represents $10 \mu \mathrm{m}$. See Methods for more details 
cytoskeleton was monitored by labeling the F-actin filaments with Alexa Fluor 546 phalloidin. Figure 1 shows maximum intensity projections of confocal images of the actin cytoskeleton of THP-1 macrophages, with progressive loss of F-actin filaments and formation of F-actin aggregates due to actin depolymerization with increasing concentrations of $\mathrm{CD}$. We did not observe any significant change in cellular morphology of THP-1 macrophages upon treatment with CD under our experimental conditions (see below). Although visual inspection of the confocal images showed a reduction in F-actin filaments upon treatment with $\mathrm{CD}$, we employed a high-resolution microscopy-based quantitative approach to get a better estimate of the loss of F-actin in macrophages (see later).

\section{Viability of THP-1 Macrophages is Not Compromised Upon Treatment with CD}

In order to quantitatively assess the effect of $\mathrm{CD}$ on the viability of THP-1 macrophages, cells were treated with CD and were tested for viability using the MTT assay, a cell proliferation assay that provides an estimate of cell growth rate and viability (Vistica et al. 1991). Figure 2 shows no significant change in the viability of macrophages upon treatment with increasing concentrations of CD relative to the control.

\section{Quantitative Estimate of F-Actin Content Upon Treatment with CD}

Intensity-based analysis is a suboptimal method to evaluate the effect of $\mathrm{CD}$ on the F-actin content of macrophages.

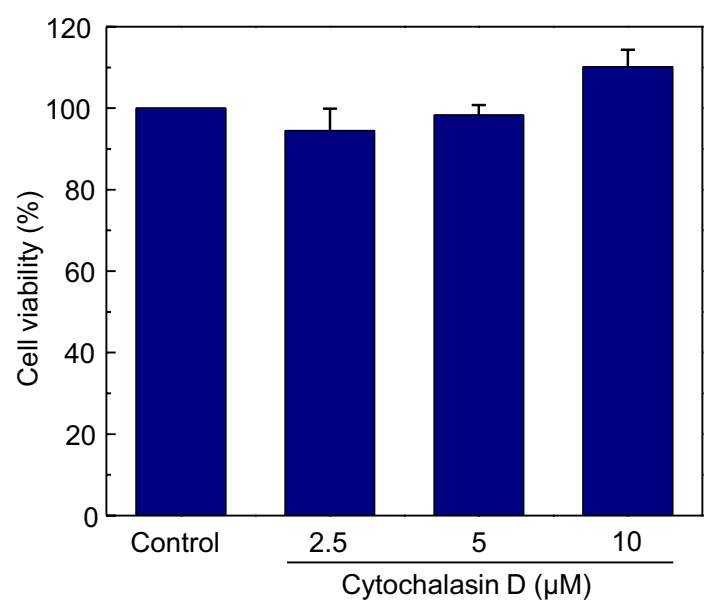

Fig. 2 Cytochalasin D has no effect on the viability of THP-1 macrophages. THP-1 macrophages were treated with increasing concentrations of CD and its effect on cell viability was assayed using MTT. Values of viability of CD-treated cells are expressed as percentages normalized to control cells. Data represent means \pm SE from at least five independent experiments. See Methods for more details
This is because F-actin aggregates that are formed due to fragmentation of actin filaments appear brighter in fluorescence microscopy relative to intact filaments. We therefore employed a high-resolution microscopy-based quantitative approach previously developed by us (Ganguly et al. 2011), to estimate changes in F-actin content in macrophages more robustly. This method works by generating iso-surface renditions from confocal z-sections of THP-1 macrophages labeled with Alexa Fluor 546 phalloidin. The volume enclosed by the iso-surfaces is normalized to the area enclosed by maximum intensity projections from confocal $\mathrm{z}$-sections of cells, thereby providing a reliable quantitative estimate of the cellular F-actin content (Ganguly et al. 2011). Figure 3A-D show iso-surface images corresponding to the projected images of macrophages treated with increasing concentrations of $\mathrm{CD}$ (see Fig. 1). Figure 3E shows a quantitative estimate of F-actin content in macrophages generated using this method and a concentration-dependent reduction in cellular F-actin levels upon treatment with CD.

\section{Actin Destabilization Inhibits Mycobacterial Entry Into Host Macrophages}

In order to probe the role of the actin cytoskeleton of host macrophages in the entry of mycobacteria, THP-1 macrophages treated with increasing concentrations of $\mathrm{CD}$ were infected with $M$. smegmatis. Following infection, colony forming units (CFU) of M. smegmatis were scored upon plating the lysate of infected macrophages and are shown in Fig. 4. The figure shows a dose-dependent reduction in the entry of M. smegmatis into host macrophages upon treatment with increasing concentrations of $\mathrm{CD}$. We observed a reduction of $\sim 30 \%$ in the entry of mycobacteria upon mild actin destabilization with $2.5 \mu \mathrm{M} \mathrm{CD}$. Upon treatment with higher concentrations ( $5 \mu \mathrm{M}$ and $10 \mu \mathrm{M})$ of $\mathrm{CD}$, we observed a progressive reduction of $\sim 40 \%$ and $\sim 50 \%$ in the entry of $M$. smegmatis into THP-1 macrophages, respectively, relative to the control (see Fig. 4). These results show that the actin cytoskeleton of host macrophages is essential for the entry of mycobacteria and any disruption of actin organization of macrophages significantly affects mycobacterial entry.

\section{Fluorescence Imaging Validates the Requirement of the Actin Cytoskeleton for the Entry of $M$. smegmatis}

We further validated the bacterial entry phenotype observed upon destabilization of the actin cytoskeleton by performing confocal microscopy of THP-1 macrophages infected with $M$. smegmatis (stably expressing dsRed 2 (Cosma et al. 2004)). Figure 5 shows representative confocal microscopy images of macrophages infected with M. smegmatis with increasing concentrations of $\mathrm{CD}$. The 

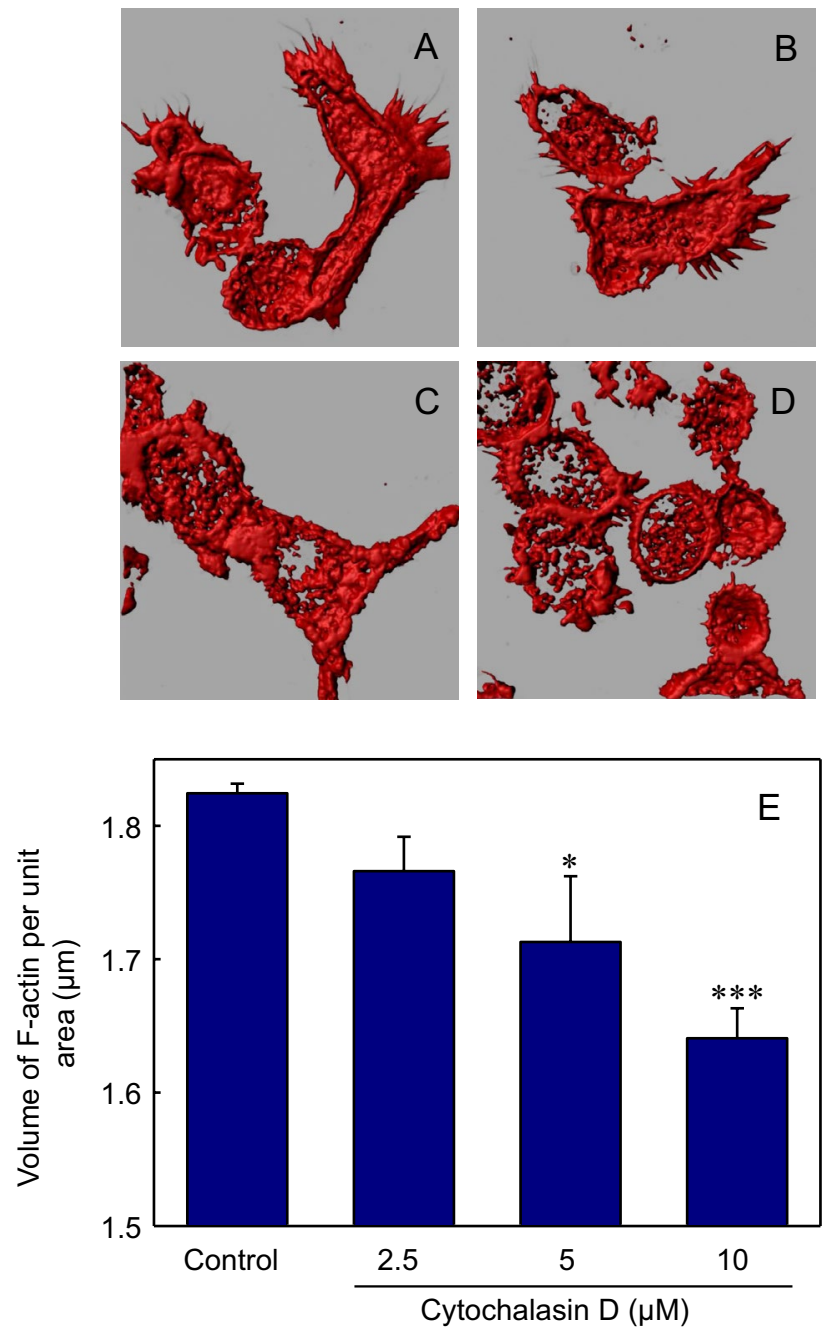

Fig. 3 Iso-surface generation and quantification of F-actin in THP-1 macrophages. Iso-surfaces of confocal $z$-sections corresponding to projections shown in Fig. 1 were generated using the iso-surface tool in Imaris. Panel (A) shows iso-surfaces for control macrophages, and iso-surfaces corresponding to macrophages treated with $2.5 \mu \mathrm{M}$, $5 \mu \mathrm{M}$ and $10 \mu \mathrm{M}$ CD are shown in panels (B-D), respectively. F-actin was quantitated by normalizing the enclosed volume within the isosurface to the projected area of cells, obtained using the Zen software provided with Zeiss LSM 880 confocal microscope. The values of F-actin content in control and CD-treated cells are shown in panel $\mathbf{E}$. Data represent means $\pm \mathrm{SE}$ of at least 15 independent measurements (* and $* * *$ correspond to significant $(p<0.05$ and $p<0.001)$ difference in F-actin content in macrophages treated with 5 and $10 \mu \mathrm{M} \mathrm{CD}$ relative to control macrophages, respectively). See Methods for more details

figure shows a concentration-dependent reduction in the entry of Mycobacterium into macrophages upon treatment with increasing concentrations of $\mathrm{CD}$, in agreement with the observations depicted in Fig. 4. These results reinforce the requirement of the actin cytoskeleton for the entry of M. smegmatis.

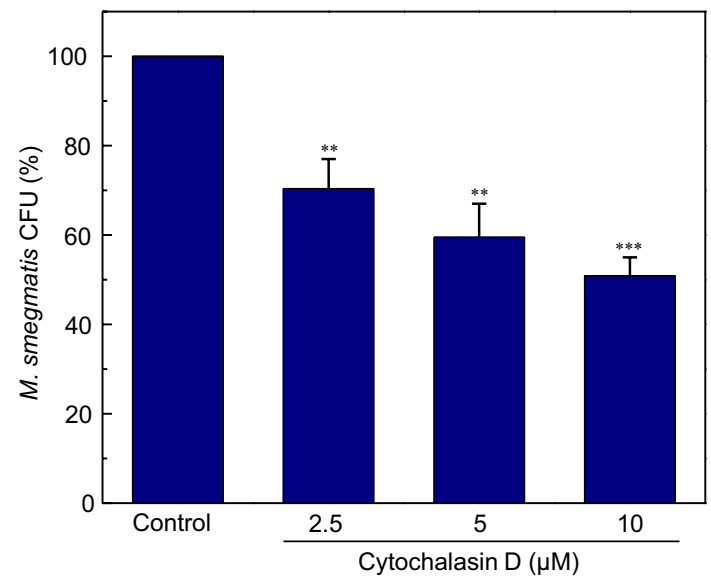

Fig. 4 Effect of actin destabilization on the entry of M. smegmatis into THP-1 macrophages. Control and CD-treated THP-1 macrophages were exposed to $M$. smegmatis at a multiplicity of infection 100:1 (bacteria to macrophage) for $2 \mathrm{~h}$. Following infection, macrophages were lysed and the intracellular bacilli were cultured on Middlebrook 7H10 agar upon making appropriate dilutions and the colony forming units (CFU) were counted. Data represent means $\pm \mathrm{SE}$ of at least six independent experiments. Values are normalized to CFU counts obtained from control (untreated) macrophages $(* *$ and $* * *$ correspond to significant $(p<0.01$ and $p<0.001)$ difference in CFU counts of CD-treated macrophages relative to control macrophages, respectively). See Methods for more details

\section{Effect of Actin Destabilization is Specific for Mycobacterial Entry}

In order to evaluate the specificity of the observed effect of actin destabilization in the entry of mycobacteria into host macrophages, we monitored the effect of $\mathrm{CD}$ treatment on the entry of Escherichia coli into THP-1 macrophages (see Fig. 6). Interestingly, the data show that the entry of $E$. coli is invariant under actin-destabilized conditions in sharp contrast to the significant reduction observed in the entry of M. smegmatis under similar conditions (see Fig. 4). These results indicate that although the entry of $M$. smegmatis is dependent on mechanisms requiring host actin cytoskeleton, the entry of $E$. coli is independent of destabilization of the actin cytoskeleton. The requirement of the intact actin cytoskeleton therefore appears to be specific for mycobacterial entry.

\section{Entry of M. smegmatis Into Host Macrophages Exhibits a Strong Correlation with Cellular F-Actin Content}

To examine a possible correlation between the reduced F-actin content of host macrophages (upon treatment with increasing concentrations of $\mathrm{CD}$ ) and the corresponding decrease in the entry of $M$. smegmatis, we plotted (shown 
Fig. 5 Confocal microscopic imaging confirms the requirement of intact host actin cytoskeleton for entry of M. smegmatis. Representative images showing the entry of M. smegmatis into control (untreated), and CD-treated macrophages. Macrophages were incubated with $M$. smegmatis stably expressing dsRed2 for $2 \mathrm{~h}$ and were processed for confocal microscopic imaging (as described in Methods). The panels show macrophages (DIC images) infected with M. smegmatis expressing dsRed2 (red), with the nucleus stained with DAPI (blue). Merged images are shown in the panel on the extreme right. The scale bar represents $10 \mu \mathrm{m}$. See Methods for more details (Color figure online)
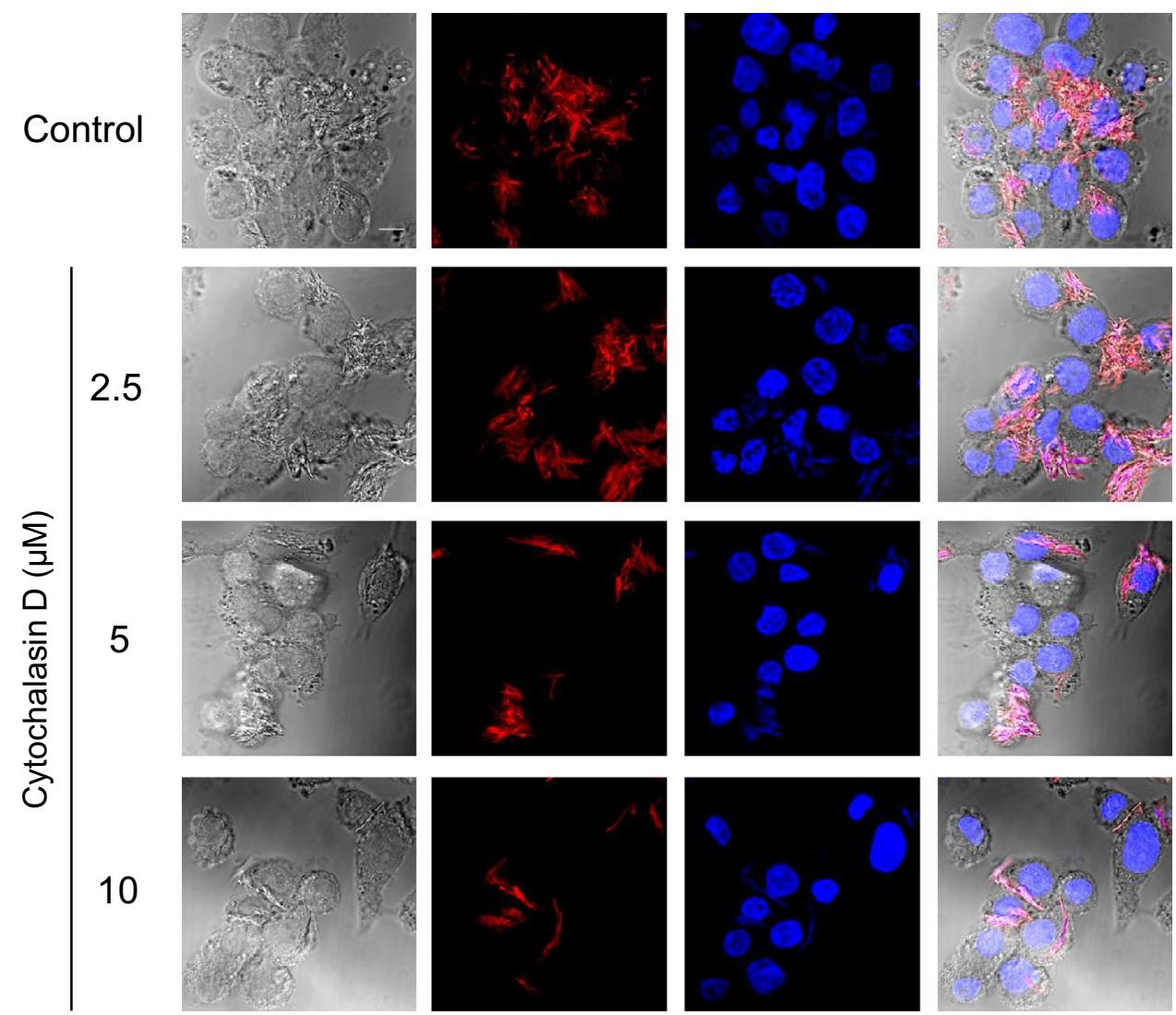

DIC

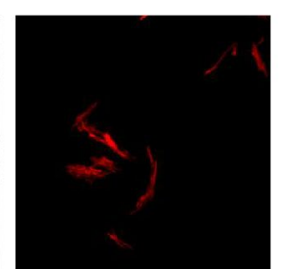

dsRed2

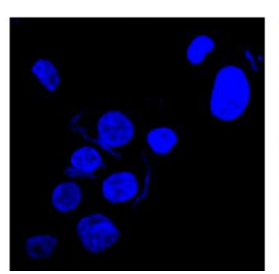

DAPI

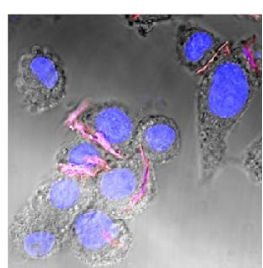

Merge

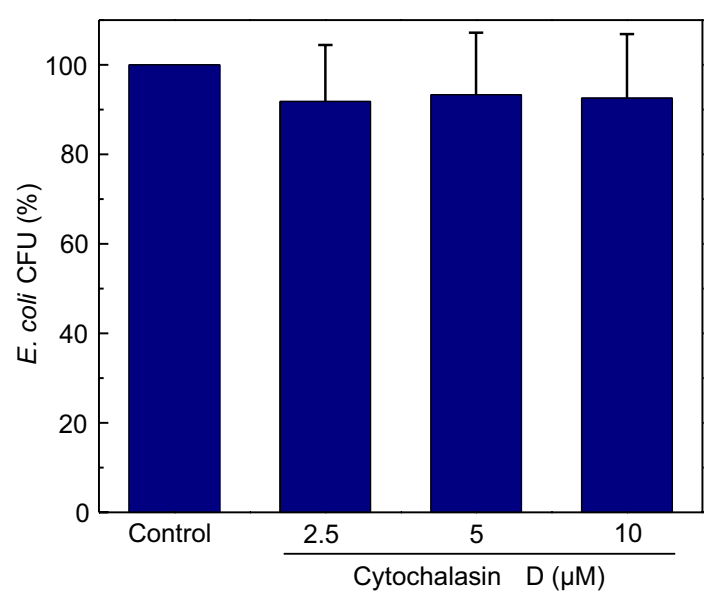

Fig. 6 Actin destabilization has no effect on the entry of E. coli into THP-1 macrophages. Control and CD-treated THP-1 macrophages were exposed to E. coli DH5 $\alpha$ at a multiplicity of infection 100:1 (bacteria to macrophage) for $2 \mathrm{~h}$. Following infection, macrophages were lysed and the intracellular bacilli were cultured on Luria Bertani agar upon making appropriate dilutions and the colony forming units (CFU) were counted. Data represent means $\pm S E$ of at least four independent experiments. Values are normalized to CFU counts obtained from control (untreated) macrophages. See Methods for more details

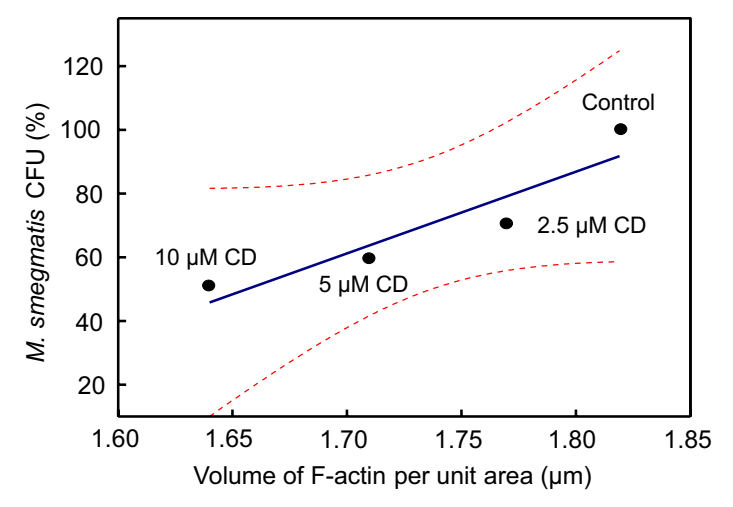

Fig. 7 Entry of M. smegmatis and host F-actin content are highly correlated. Correlation of $M$. smegmatis $\mathrm{CFU}$ counts and F-actin content of host macrophages upon treatment with increasing concentrations of CD. Data for the figure are from Figs. 3 and 4. Linear regression analysis yielded a correlation coefficient $(r)$ of $\sim 0.93$. All data points were bound by the $95 \%$ confidence band (plotted as red dashed lines), implying a significant dependence of mycobacterial entry on the host actin cytoskeleton. See Methods and text for further details (Color figure online)

in Fig. 7) levels of cellular F-actin (from Fig. 3E) vs. CFU counts of $M$. smegmatis (from Fig. 4). Figure 7 shows a linear regression analysis of F-actin levels of macrophages and the CFU counts of $M$. smegmatis which yielded a positive 
correlation of $\sim 0.93$. The figure shows that all the data points are bound by the $95 \%$ confidence band, which implies a significant dependence of mycobacterial entry on host actin cytoskeleton.

\section{Discussion}

The actin cytoskeleton constitutes a dynamic protein framework within cells that has been shown to be involved in essential functions including cellular mobility, trafficking and signaling (Janmey 1998). An important emerging feature associated with the organization of actin involves its intimate association and interplay with the cellular plasma membrane. Such an interplay has been implicated in functional outcomes such as regulation of membrane stiffness, morphological polarity and migration of cells, and dynamics of G protein-coupled receptors (GPCRs) (Pierini et al. 2003; Byfield et al. 2004; Tsai et al. 2006; Sun et al. 2007; Ganguly and Chattopadhyay 2010). In view of the fact that the host plasma membrane serves as the first portal of entry for intracellular pathogens to gain access into the host cell interior (Kumar et al. 2016), these observations on actinmembrane interplay make the exploration of the role of the actin cytoskeleton in the entry of intracellular pathogens extremely relevant. From this perspective, our results demonstrating the role of the integrity of host actin cytoskeleton for mycobacterial entry assume significance. To the best of our knowledge, these results constitute one of the first reports employing a quantitative microscopy-based approach to explore the effect of actin reorganization in mycobacterial entry. The close correlation exhibited by these two processes further enhances the significance underlying such observed effects.

In an earlier study, we demonstrated the requirement of host actin cytoskeleton in the entry of the protozoan parasite Leishmania donovani (Roy et al. 2014). Although there are reports on Mycobacterium-induced changes in the organization of intracellular actin, especially in the context of the maturation of pathogen-containing phagosomes and their fusion with lysosomes (Guérin and de Chastellier 2000; Hestvik et al. 2005), direct evidence on the role of the host actin cytoskeleton in the entry of mycobacteria into their hosts is lacking, and the precise mechanism by which Mycobacteria enter host cells remains poorly understood. While in vitro studies have identified roles for specific receptors in internalization (Ernst 1998; Killick et al. 2013; Awuh and Flo 2017), it is plausible that Mycobacteria could engage a combination of receptors for their entry, depending on cellular identity. Mycobacterial uptake into epithelial cells, a non-phagocytic cell type, has been shown to require actin cytoskeleton rearrangement, which is regulated by ADPribosylation factor 1 and phospholipase D1, and is dependent on the M3 muscarinic receptor (Song et al. 2018). It is conceivable that the entry defect we observe on inhibition of actin polymerization reflects a compromise in the endocytic pathway involved in internalization. Exploring if this defect lies in a specific endocytic pathway (such as clathrin- or caveolin-mediated phagocytosis) as has been described by us for the entry of Leishmania donovani into murine macrophages (Kumar et al. 2019) could potentially open avenues for the development of therapeutic approaches targeting the specific route of entry.

With an overall objective to explore the role of the host plasma membrane in the entry of mycobacteria, we have recently shown that $M$. smegmatis requires an optimum level of host membrane cholesterol for efficient entry into host cells (Viswanathan et al. 2015). Cholesterol depletion from membranes has been shown to induce changes in the underlying actin cytoskeleton by loss or redistribution of phosphatidylinositol-4,5-bisphosphate (Kwik et al. 2003). In addition, cholesterol depletion could affect membrane rigidity and neutrophil migration in a manner dependent on the polymeric state of actin (Pierini et al. 2003; Byfield et al. 2004). Although the mechanistic basis underlying the interplay between the actin cytoskeleton and membrane cholesterol remains a subject of active investigation (Sarkar P, Kumar GA, Shrivastava S, Chattopadhyay A, unpublished observations), the consequences of such an interplay in active membrane-associated events such as pathogen entry cannot be ruled out. In addition, we demonstrated that metabolic inhibition of host sphingolipid synthesis leads to reduction in the entry of $M$. smegmatis into host cells (Viswanathan et al. 2018). We believe that a comprehensive understanding of key membrane components encompassing membrane lipids such as cholesterol and sphingolipids, along with the underlying actin cytoskeleton, would provide a comprehensive molecular insight into the process of mycobacterial entry.

The entry of mycobacteria involves host membrane proteins such as complement receptors, mannose receptor, CD-14, scavenger receptors, DC-SIGN, and dectin-1 that act as receptors for pathogen recognition (Ernst 1998; Killick et al. 2013; Awuh and Flo 2017). In addition, a recent study has reported that by manipulating GPCRs in host cells, mycobacteria could suppress host cell signaling pathways (Alaridah et al. 2017). In this overall context, previous work from our group has highlighted the role of the actin cytoskeleton in the function and dynamics of GPCRs (Ganguly et al. 2008; Shrivastava et al. 2020). The ability of the actin cytoskeleton to modulate function of membrane proteins involved in pathogen recognition by host cells could provide a molecular basis underlying the role of the actin cytoskeleton in mycobacterial entry. Taken together along with our previous work on Leishmania donovani (Roy et al. 2014), our present results on M. smegmatis highlight 
a potential common theme involving the role of the host actin cytoskeleton in the entry of intracellular pathogens. We envision that our results could assume significance in developing novel therapeutic strategies targeting actin-mediated mycobacterial entry in host cells.

The entry of intracellular pathogens into host cells is dependent on multiple factors, most important of these being the interaction between pathogenic and host molecules at the plasma membrane. In the context of the emerging concept of the interplay between membrane cholesterol and the actin cytoskeleton, and in view of our previous observations on the role of cholesterol in mycobacterial entry, our results (using a robust quantitative confocal microscopy-based technique) on the role of actin cytoskeleton in the entry of mycobacteria into human macrophages assume significance. Our results comprehensively demonstrate a link between actin organization and efficiency of mycobacterial entry, with reduced levels of F-actin correlating with a significant reduction in pathogen internalization. We believe that, in addition to cholesterol and sphingolipids, these results now add a third factor intimately associated with the plasma membrane as a major player in mycobacterial entry into macrophages. This opens up an additional point of possible therapeutic intervention targeting host entry as an anti-TB strategy, which has important connotations in the context of the alarming rise in $M$. $t b$ infections resistant to the currently available antibiotics that target the pathogen.

Acknowledgements This work was supported by a Council of Scientific and Industrial Research (CSIR) Focused Biology Research (FBR) grant to T.R.R. and A.C. (MLP 0144). A.D. and R.P.M. thank the Council of Scientific and Industrial Research (CSIR) and Department of Biotechnology (DBT) for the award of Research Associateship and Senior Research Fellowship, respectively. G.A.K. was supported as a Senior Project Associate by a CSIR FBR grant to A.C. (MLP 0146). AC gratefully acknowledges support from SERB Distinguished Fellowship and CSIR Bhatnagar Fellowship. We thank members of the Chattopadhyay laboratory and Bhagyashree Rao for critically reading the manuscript and for their comments and suggestions.

Author Contributions MJ, TRR and AC conceptualized the project; $\mathrm{AD}, \mathrm{MJ}, \mathrm{RPM}$ and GAK performed experiments and analyzed data; GAK, MJ, TRR and AC designed experiments; AD, MJ, GAK and AC wrote the manuscript; TRR and AC edited the manuscript, organized access to research facilities and funding, and provided overall supervision and mentoring.

\section{Declarations}

Conflict of Interest The authors declare that they have no conflicts of interest.

\section{References}

Alaridah N, Lutay N, Tenland E, Rönnholm A, Hallgren O, Puthia M, Westergren-Thorsson G, Godaly G (2017) Mycobacteria manipulate G-protein-coupled receptors to increase mucosal Rac1 expression in the lungs. J Innate Immun 9:318-329

Allué-Guardia A, García JI, Torrelles JB (2021) Evolution of drugresistant Mycobacterium tuberculosis strains and their adaptation to the human lung environment. Front Microbiol 12:612675

Asrat S, de Jesús DA, Hempstead AD, Ramabhadran V, Isberg RR (2014) Bacterial pathogen manipulation of host membrane trafficking. Annu Rev Cell Dev Biol 30:79-109

Awuh JA, Flo TH (2017) Molecular basis of mycobacterial survival in macrophages. Cell Mol Life Sci 74:1625-1648

Bagga S, Bouchard MJ (2014) Cell cycle regulation during viral infection. Methods Mol Biol 1170:165-227

Byfield FJ, Aranda-Espinoza H, Romanenko VG, Rothblat GH, Levitan I (2004) Cholesterol depletion increases membrane stiffness of aortic endothelial cells. Biophys J 87:3336-3343

Chakrabarti R, Lee M, Higgs HN (2021) Multiple roles for actin in secretory and endocytic pathways. Curr Biol 31:R603-R618

Chaurasiya SK (2018) Tuberculosis: smart manipulation of a lethal host. Microbiol Immunol 62:361-379

Cosma CL, Humbert O, Ramakrishnan L (2004) Superinfecting mycobacteria home to established tuberculous granulomas. Nat Immunol 5:828-835

Dominguez R, Holmes KC (2011) Actin structure and function. Annu Rev Biophys 40:169-186

Dye C (2009) Doomsday postponed? preventing and reversing epidemics of drug-resistant tuberculosis. Nat Rev Microbiol 7:81-87

El-Etr SH, Cirillo JD (2001) Entry mechanisms of mycobacteria. Front Biosci 6:737-747

Ernst JD (1998) Macrophage receptors for Mycobacterium tuberculosis. Infect Immun 66:1277-1281

Fraga AG, Barbosa AM, Ferreira CM, Fevereiro J, Pedrosa J, Torrado E (2018) Immune-evasion strategies of mycobacteria and their implications for the protective immune response. Curr Issues Mol Biol 25:169-198

Gandhi NR, Moll A, Sturm AW, Pawinski R, Govender T, Lalloo U, Zeller K, Andrews J, Friedland G (2006) Extensively drugresistant tuberculosis as a cause of death in patients co-infected with tuberculosis and HIV in a rural area of South Africa. Lancet 368:1575-1580

Ganguly S, Chattopadhyay A (2010) Cholesterol depletion mimics the effect of cytoskeletal destabilization on membrane dynamics of the serotonin ${ }_{1 \mathrm{~A}}$ receptor: a zFCS study. Biophys J 99:1397-1407

Ganguly S, Pucadyil TJ, Chattopadhyay A (2008) Actin cytoskeleton dependent dynamics of the serotonin $n_{1 \mathrm{~A}}$ receptor correlates with receptor signaling. Biophys J 95:451-463

Ganguly S, Saxena R, Chattopadhyay A (2011) Reorganization of the actin cytoskeleton upon G-protein coupled receptor signaling. Biochim Biophys Acta 1808:1921-1929

Guérin I, de Chastellier C (2000) Disruption of the actin filament network affects delivery of endocytic contents marker to phagosomes with early endosome characteristics: the case of phagosomes with pathogenic mycobacteria. Eur J Cell Biol 79:735-749

Hestvik ALK, Hmama Z, Av-Gay Y (2005) Mycobacterial manipulation of the host cell. FEMS Microbiol Rev 29:1041-1050

Honigmann A, Pralle A (2016) Compartmentalization of the cell membrane. J Mol Biol 428:4739-4748

Janmey PA (1998) The cytoskeleton and cell signaling: component localization and mechanical coupling. Physiol Rev 78:763-781

Keshavjee S, Farmer PE (2012) Tuberculosis, drug resistance, and the history of modern medicine. N Engl J Med 367:931-936 
Killick KE, Ní Cheallaigh C, O'Farrelly C, Hokamp K, MacHugh DE, Harris J (2013) Receptor-mediated recognition of mycobacterial pathogens. Cell Microbiol 15:1484-1495

Kumar GA, Jafurulla M, Chattopadhyay A (2016) The membrane as the gatekeeper of infection: cholesterol in host-pathogen interaction. Chem Phys Lipids 199:179-185

Kumar GA, Karmakar J, Mandal C, Chattopadhyay A (2019) Leishmania donovani internalizes into host cells via caveolin-mediated endocytosis. Sci Rep 9:12636

Kwik J, Boyle S, Fooksman D, Margolis L, Sheetz MP, Edidin M (2003) Membrane cholesterol, lateral mobility, and the phosphatidylinositol 4,5-bisphosphate-dependent organization of cell actin. Proc Natl Acad Sci USA 100:13964-13969

Lange C, Chesov D, Heyckendorf J, Leung CC, Udwadia Z, Dheda K (2018) Drug-resistant tuberculosis: An update on disease burden, diagnosis and treatment. Respirology 23:656-673

Liu CH, Liu H, Ge B (2017) Innate immunity in tuberculosis: host defense vs pathogen evasion. Cell Mol Immunol 14:963-975

Pierini LM, Eddy RJ, Fuortes M, Seveau S, Casulo C, Maxfield FR (2003) Membrane lipid organization is critical for human neutrophil polarization. J Biol Chem 278:10831-10841

Roy S, Kumar GA, Jafurulla M, Mandal C, Chattopadhay A (2014) Integrity of the actin cytoskeleton of host macrophages is essential for Leishmania donovani infection. Biochim Biophys Acta 1838:2011-2018

Sampath P, Pollard TD (1991) Effects of cytochalasin, phalloidin, and $\mathrm{pH}$ on the elongation of actin filaments. Biochemistry 30:1973-1980

Sani M, Houben EN, Geurtsen J, Pierson J, de Punder K, van Zon M, Wever B, Piersma SR, Jiménez CR, Daffé M, Appelmelk BJ, Bitter W, van der Wel N, Peters PJ (2010) Direct visualization by cryo-EM of the mycobacterial capsular layer: a labile structure containing ESX-1-secreted proteins. PLoS Pathog 6:e1000794

Schliwa M (1982) Action of cytochalasin D on cytoskeletal networks. J Cell Biol 92:79-91

Shrivastava S, Sarkar P, Preira P, Salomé L, Chattopadhyay A (2020) Role of actin cytoskeleton in dynamics and function of the serotonin $_{1 \mathrm{~A}}$ receptor. Biophys J 118:944-956

Singh R, Dwivedi SP, Gaharwar US, Meena R, Rajamani P, Prasad $\mathrm{T}$ (2020) Recent updates on drug resistance in Mycobacterium tuberculosis. J Appl Microbiol 128:1547-1567

Song OR, Queval CJ, Iantomasi R, Delorme V, Marion S, VeyronChurlet R, Werkmeister E, Popoff M, Ricard I, Jouny S, Deboosere N (2018) Arf GAP 1 restricts Mycobacterium tuberculosis entry by controlling the actin cytoskeleton. EMBO Rep 19:29-42
Stanley SA, Cox JS (2013) Host-pathogen interactions during Mycobacterium tuberculosis infections. Curr Top Microbiol Immunol 374:211-241

Sun M, Northup N, Marga F, Huber T, Byfield FJ, Levitan I, Forgacs G (2007) The effect of cellular cholesterol on membrane-cytoskeleton adhesion. J Cell Sci 120:2223-2231

Thakur A, Mikkelsen H, Jungersen G (2019) Intracellular pathogens: host immunity and microbial persistence strategies. J Immunol Res 2019:1356540

Tiwari BM, Kannan N, Vemu L, Raghunand TR (2012) The Mycobacterium tuberculosis PE proteins Rv0285 and Rv1386 modulate innate immunity and mediate bacillary survival in macrophages. PLoS One 7:e51686

Tiwari B, Soory A, Raghunand TR (2014) An immunomodulatory role for the Mycobacterium tuberculosis region of difference 1 locus proteins PE35 (Rv3872) and PPE68 (Rv3873). FEBS J 281:1556-1570

Tsai H-I, Tsai L-H, Chen M-Y, Chou Y-C (2006) Cholesterol deficiency perturbs actin signaling and glutamate homeostasis in hippocampal astrocytes. Brain Res 1104:27-38

Vistica DT, Skehan P, Scudiero D, Monks A, Pittman A, Boyd MR (1991) Tetrazolium based assays for cellular viability: a critical examination of selected parameters affecting formazan production. Cancer Res 51:2515-2520

Viswanathan G, Jafurulla M, Kumar GA, Raghunand TR, Chattopadhyay A (2015) Dissecting the membrane cholesterol requirement for mycobacterial entry into host cells. Chem Phys Lipids 189:19-27

Viswanathan G, Jafurulla M, Kumar GA, Raghunand TR, Chattopadhyay A (2018) Macrophage sphingolipids are essential for the entry of mycobacteria. Chem Phys Lipids 213:25-31

World Health Organization (2021) 2021: Global tuberculosis report 2021. https://www.who.int/news-room/fact-sheets/detail/tuber culosis; https://www.who.int/teams/global-tuberculosis-progr amme/covid-19

Zhang J (2013) Transcriptome analysis reveals novel entry mechanisms and a central role of SRC in host defense during high multiplicity mycobacterial infection. PLoS One 8:e65128

Publisher's Note Springer Nature remains neutral with regard to jurisdictional claims in published maps and institutional affiliations.

\section{Authors and Affiliations}

\section{Aritri Dutta $^{1} \cdot$ Ravi Prasad Mukku ${ }^{1}$. G. Aditya Kumar ${ }^{1,2} \cdot$ Md. Jafurulla $^{1} \cdot$ Tirumalai R. Raghunand $^{1}$. Amitabha Chattopadhyay ${ }^{1}$ (B)}

Tirumalai R. Raghunand

raghu@ccmb.res.in

Amitabha Chattopadhyay

amit@ccmb.res.in
CSIR-Centre for Cellular and Molecular Biology, Uppal Road, Hyderabad 500007, India

2 Department of Pharmacology, University of Michigan Medical School, Ann Arbor, MI 48109, USA 